\title{
Multiparasitic Infection (Hydatid Cyst of the Spleen and Ascaris Lumbricoides Infestation) Mimicking an Acute Surgical Abdomen - Case Report
}

\author{
Molnar Călin ${ }^{1}$, Vizitiu Alexandru1*, Deak Karoly¹, Russu Cristian¹, Tudor Adrian¹, Suciu Bogdan ${ }^{1}$, Stolnicu \\ Simona², Hulub Marius³, Molnar Claudiu4 \\ 1 Surgical Clinic No. I, Clinical Emergency County Hospital Tirgu Mures, University of Medicine and Pharmacy Tirgu Mures, Romania \\ 2 Anathomo-Pathology Clinic, Clinical Emergency County Hospital Tirgu Mures, University of Medicine and Pharmacy Tirgu Mures, Romania \\ 3 University of Medicine and Pharmacy Tirgu Mures, Romania \\ ${ }^{4}$ Obstetric-Gynecology Clinic, Clinical Emergency County Hospital Tirgu Mures, University of Medicine and Pharmacy Tirgu Mures, Romania
}

Hydatid cyst involving the spleen is a rare clinical condition (0.5-4\%). We report a case of multi-parasitic infection in a 62 year old female (hydatid cyst and ascaris lumbricoides infestation), confirmed during surgery. The purpose of the paper is to emphasize on the rare association of the two parasitic infections, affecting two different organs (spleen and jejunum). The combined symptomatology of the two parasites could mimic an acute surgical abdomen.

Keywords: multiparasitic infection, hydatid cyst of the spleen, ascarisis

Received: 23 August 2015 / Accepted: 02 November 2015

\section{Introduction}

First reported by Berlot in 1790 during an autopsy, the splenic hydatid cyst is an extremely rare disease, representing only $0.5-4 \%$ of the primary infestations. It is an endemic disease in sheep and cattle raising countries from South America, Africa, Asia, South Europe and India, with the highest incidence in Iran [1]. In Romania the incidence of hydatidosis is about 5 cases/ 100.000 people [2].

The most common organ involved in hydatid disease is the liver $(75 \%)$, followed by the lung (15\%). The primary involvement of the spleen is exceptional (2.5\%) [3]. The primary infestation of the spleen usually takes place by arterial route, after the parasite passes two filters: hepatic and pulmonary. There is reported a venous route of infestation, through the splenic vein (portal venous system) in patients with portal hypertension [4].

Ascaris lumbricoides nematatodes infect over 1 bilion people annually [5]. Ascarisis is the most common soiltransmitted heminthes infestation. The parasite matures in the small intestine; most of the parasites are expelled on the $23^{\text {rd }}$ week [6]. There is very little data about this combined parasitosis, hydatid cyst of the spleen and ascarisis. Also, there are no described cases in which the patient's symptomatology mimics an acute surgical abdomen.

\section{Case report}

We report the case of a 62 year old woman, from the rural area, admitted in the Surgery I Clinic from the Emergency County Hospital Tirgu Mures. She presented with a gigantic lump in the left hypochondrium associated with

* Correspondence to: Alexandru Vizitiu

E-mail: dr_vizi@yahoo.com pain. On admission her general status was influenced by the abdominal pain, mimicking an acute surgical abdomen. From her history we found out that the lump appeared about 2 years ago and gradually increased in size. We observe an altered general status, fatigue, dyspnea, pain in the left half of the abdomen, flatulence with the absence of intestinal transit, nausea and vomiting. From her past medical history we remark hydatid phthisic, kidney failure, a renal cyst and sinus tachycardia. Examination of the abdomen reveals an expanded abdomen, deformed by a gigantic lump in the left hypochondrium $(36 \times 31 \times 31)$, which limits the respiratory movements. The lump was painful on palpation, of firm consistency, oval in shape, had a smooth surface, immobile, with muscular defense present on deep palpation in the region (Figure 1). On auscultation we observed hyperactive peristaltic sounds. The gynecologic examination was unremarkable. Her routine hematological and biochemical investigations were normal (no eosinophilia). The chest X-Ray came back normal as well, without pulmonary hydatid cysts. Abdominal CT scan reveals a gigantic tumor in the left hipochondrium (40x30x30) with many septa which incorporates the spleen, which could plead for a hydatid cyst of the spleen. The tumor displaces the stomach to the right and, caudally the transverse and left colon.

Considering the altered general status we discarded the preoperative antiparasitic treatment and proceeded with the surgical intervention. The procedure took place under general anesthesia. A left subcostal laparotomy, extended to the right - á la LeClerc (Figure 2) was performed. Surgical exploration revealed a gigantic tumor of approximate $35 \times 30 \times 30 \mathrm{~cm}$ that pushes the stomach to the right and the left kidney infero-medialy, delimited by the mezoco- 


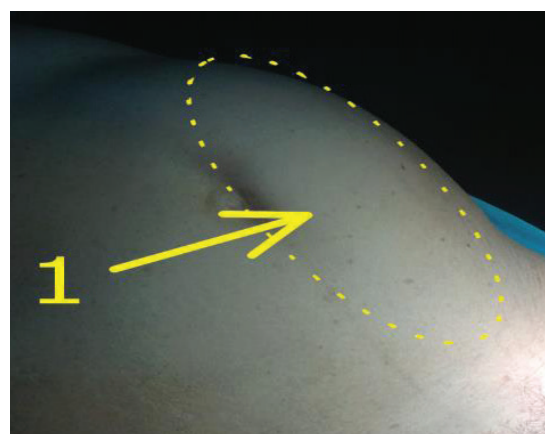

Fig. 1. Clinical aspect of abdomen

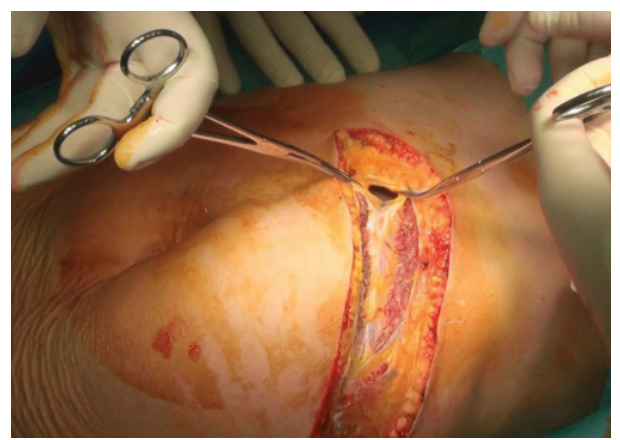

Fig. 2. Left LeClerc incision

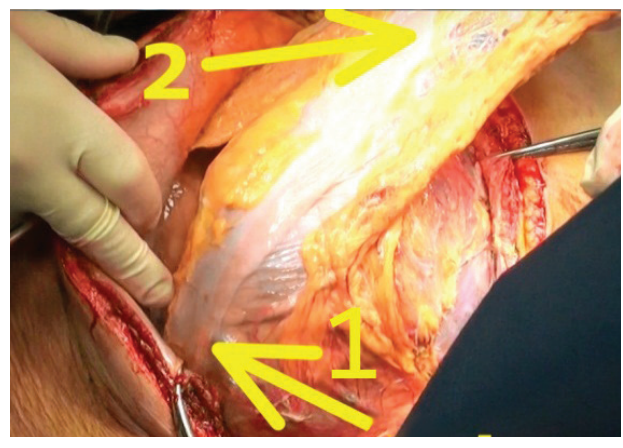

Fig. 3. Tumoral aspects: (1) transverse colon; (2) left colon lon and splenic flexure of the colon (Figures 3 and 4). The tumor was adherent to the diaphragm. We detached the colon with the Ligasure Impact and Ligasure Atlas devices (Figure 5) and punctioned the cystic tumor to avoid dissemination in the peritoneal cavity (on puncture we evacuate a clear fluid). In order to treat any potential contamina-

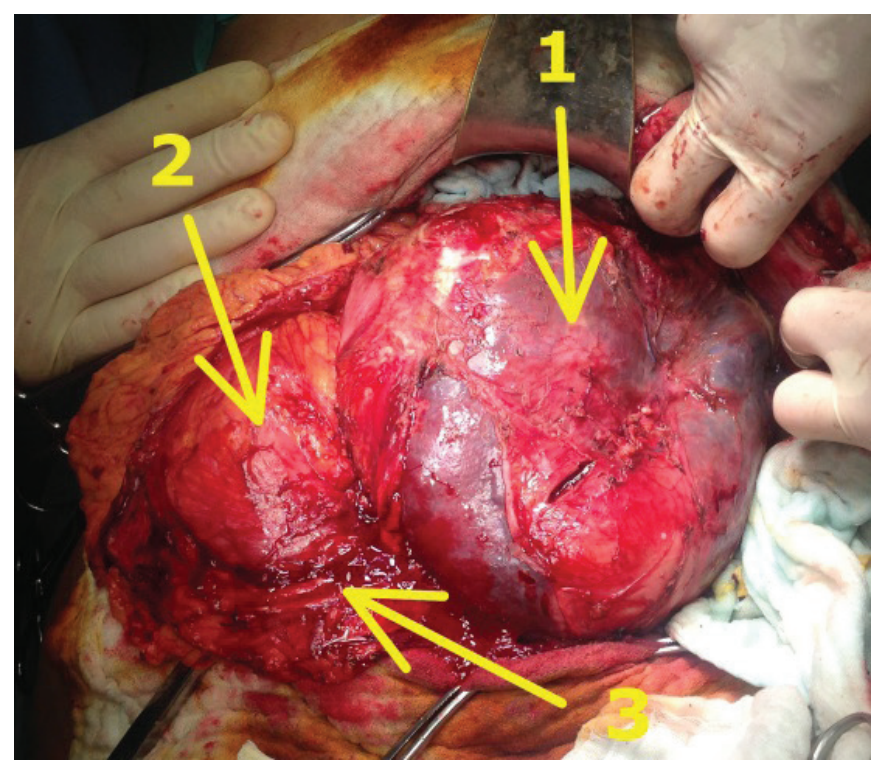

Fig. 4. Tumoral aspects: (1) splenic tumor formation; (2) stomach; (3) transverse colon

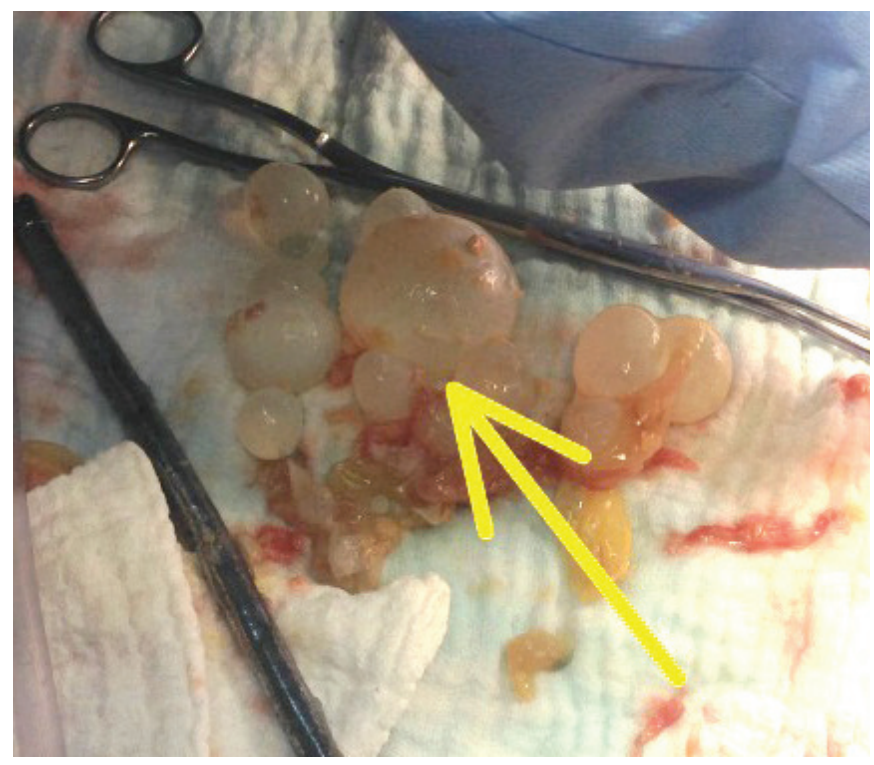

Fig. 6. Hidatic live elements: daughter cysts and vesicles (65) tion we apply isolations soaked in alcohol. We evacuate the cyst wall, encircling many solices. On our section there was around 5 litres of hydatid fluid (Figure 6). A total splenectomy was performed, with the help of the Ligasure devices, taking special care of the tail of the pancreas and splenic flexure of the colon (Figures 7 and 8). Systematic explora-

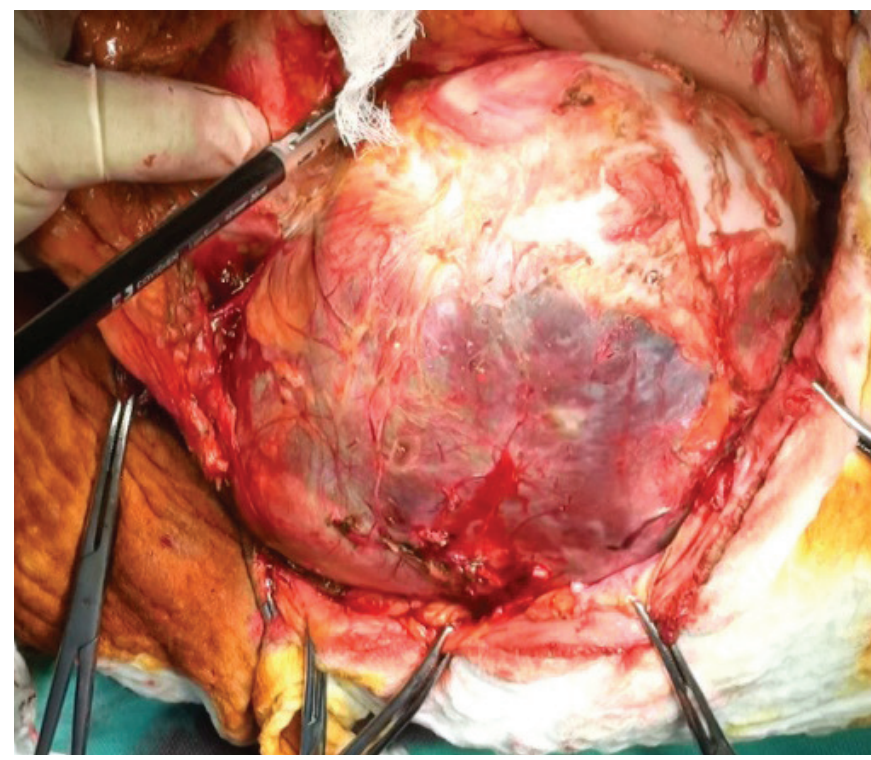

Fig. 5. Colo-epiploic detachment

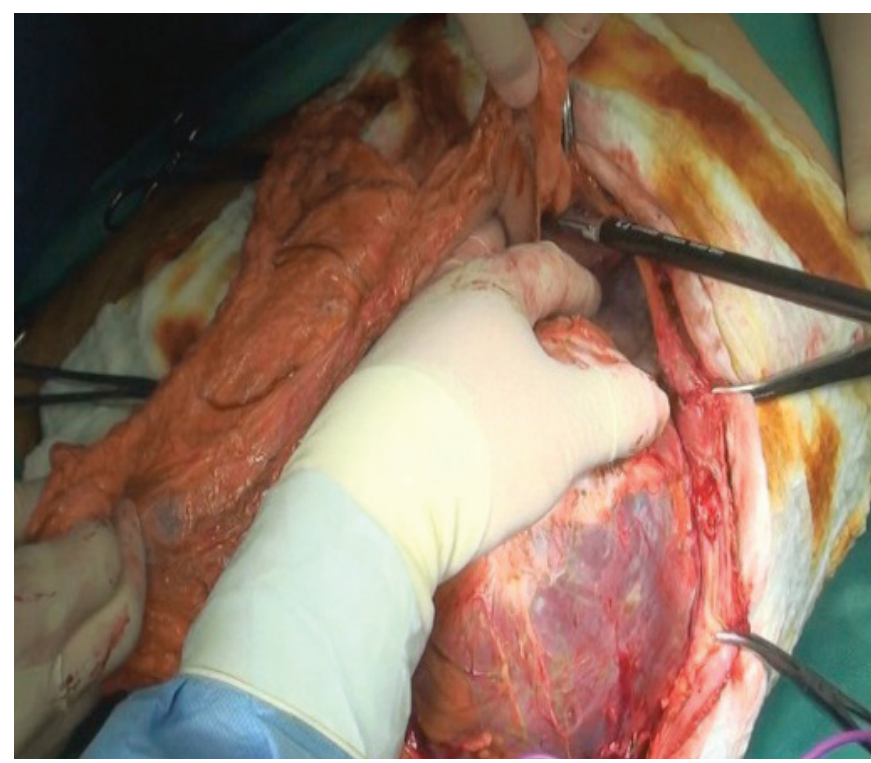

Fig. 7. Sparing the pancreas 


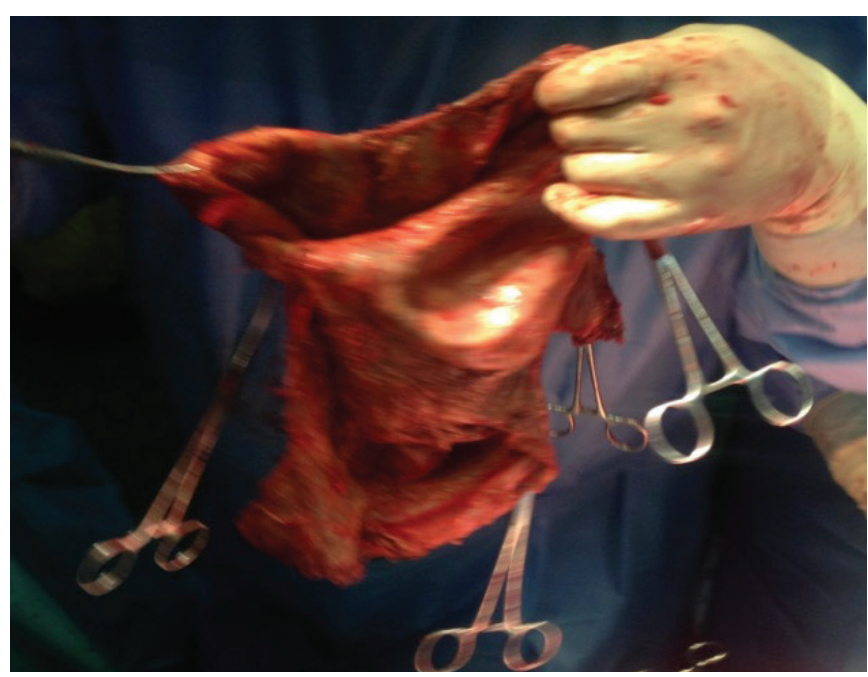

Fig. 8. Ideal total histo-pericystectomy

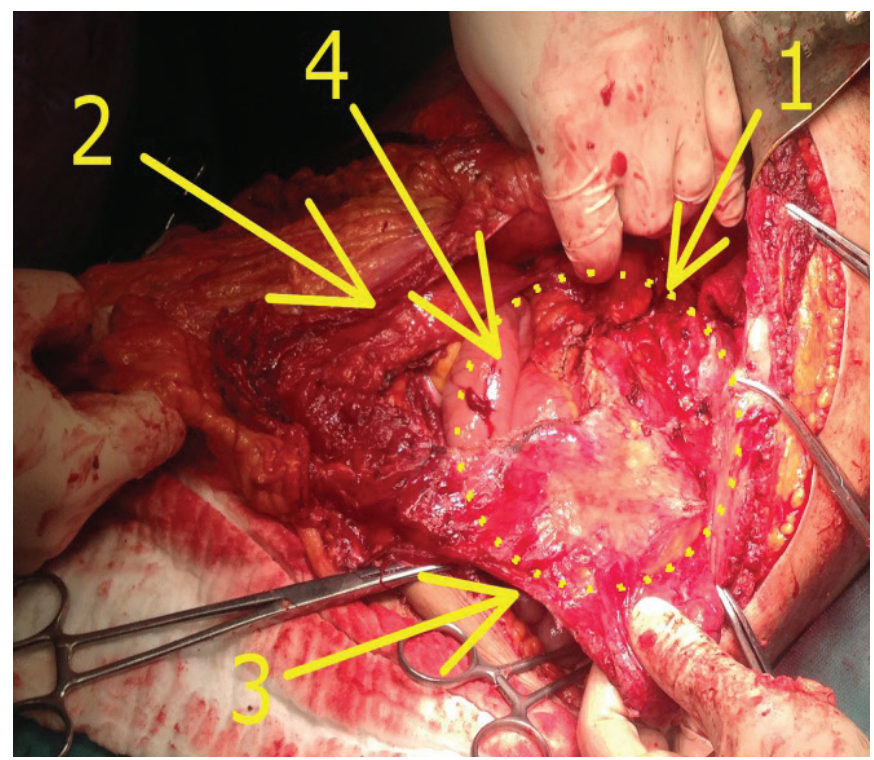

Fig. 10. Remanent cavity. (1) post pericystectomy cavity; (2) duodenum; (3) jejunum; (4) mesenterium

tion of the peritoneal cavity revealed the concurrent infection with ascaris lumbricoides (two adult parasites in the proximal jejunum) (Figure 9) but no surgical act was taken because of the known complication of fistulae in these type of infections (Figures 10 and 11). Postoperative treatment with Albendazol 2x400 mg/day was prescribed as soon as normal bowel movement is reestablished. The postoperative course was uneventful, the patient being discharged on the $7^{\text {th }}$ day with additional Albendazole treatment.

\section{Discussions}

The splenic hydatid cyst has a slow development of 1-2 $\mathrm{cm} /$ year in diameter [7]. The latent period of cyst development is usually long: 5 to 20 years. Splenic hydatid cysts develop insidiously and may reach a large size. Clinical manifestations of hydatid splenic disease are usually mild and non-specific. The main symptom in gigantic cysts [8] is abdominal pain associated to a dyspeptic syndrome and weight loss [7,9].

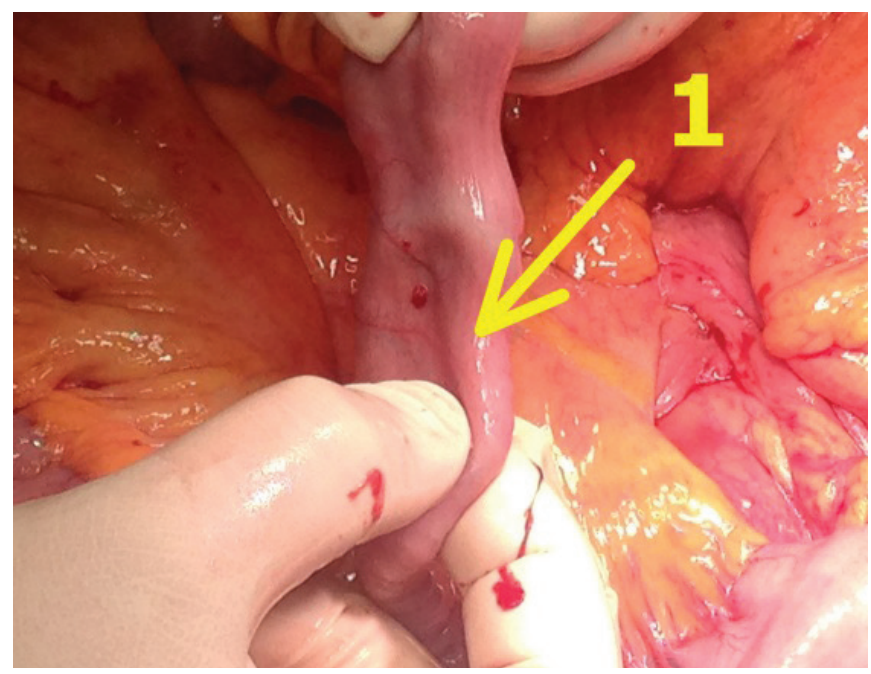

Fig. 9. Intrajejunal adult Ascaris lumbricoides

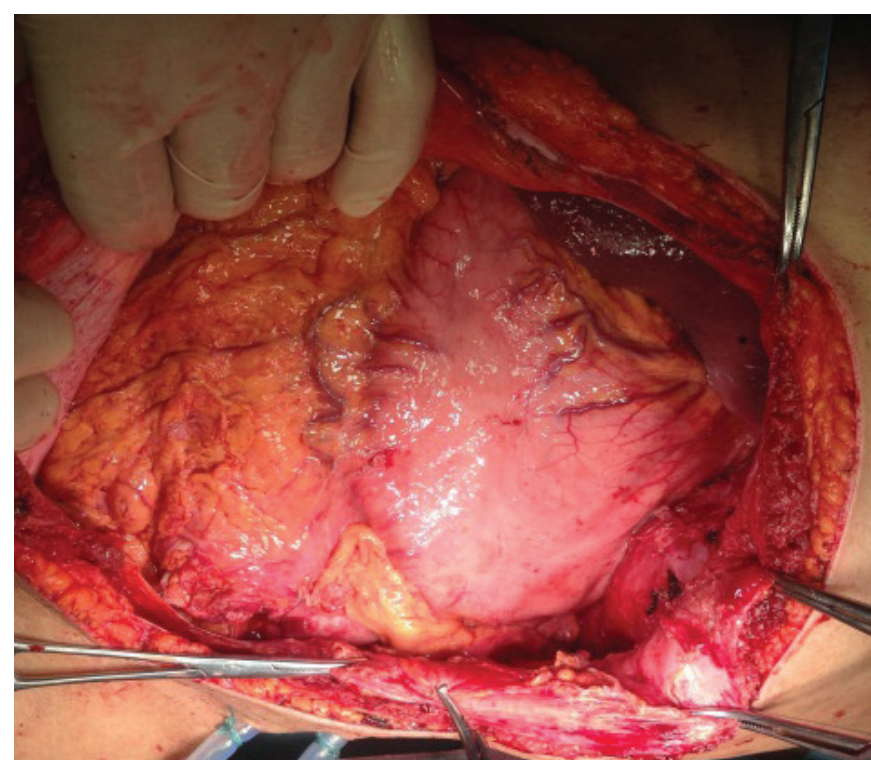

Fig. 11. Final aspect after procedure

Main differential diagnoses of splenic hydatidosis are splenic cystic lesions such as dermoid cyst, pseudocyst, abscess, intrasplenic pancreatic pseudocyst, pseudocyst from splenic trauma, infarcting infection, haematoma, metastatic disease and cystic lymphangioma or hemangioma. If eosinophilia is present, other parasitosis may be taken into account $[10,11]$.

Symptoms like: abdominal pain, loss of appetite, absence of bowel movement, nausea, vomiting, fatigue are unspecific and common for both parasitic infestations (hydatidosis and ascarisis) [12].

Total splenectomy is the preffered approach undertaken in cases with large, multiple and symptomatic cysts, with the removal of all the parasitic tissue. Partial splenectomy, cyst enucleation or omentoplasty after partial resection of the cyst wall are surgical options for the treatment of splenic hydatidosis, but these techniques are used for superficial cysts, cysts localized in one pole of the spleen or cysts that are unresectable due to extensive adhesions. Par- 
tial splenectomy is a hemorrhagic intervention because it is difficult to have vascular control when incising the splenic tissue, whereas unroofing the cyst wall leaves behind the pericystic layer and a residual cavity which carries the risk of postoperative infection [13].

It is important that we mention the contribution of the Ligasure devices. Using them we had the possibility to approach hidden anatomic spaces, making resections and in the same time hemostasis. It is also worth mentioning that we used these devices without any risks in flammable substances (the alcohol used to neutralize the parasites).

The main control strategy against soil-transmitted helminthes is the medical treatment with Albendazole, being cost-efficient and easy to administer [14].

The association of two parasitic infections is rare and usually found in immunocompromised patients with poor social status. Infestation with helminthes is usually a intraoperative finding.

\section{Conclusions}

The multiparasitic infection, with ascaris lumbricoides and splenic hydatidosis, which together mimic an acute surgical abdomen gives this case report unicity.

The antiparasitic treatment is the most important measure to treat a parasitosis. Regarding the surgical intervention, it must be carefully selected in cases where the patient's status allows it, being considered a second line treatment.

\section{Conflict of interest}

None to declare

\section{References}

1. Khalid Rasheed, Showkat Ali Zargar, and Ajaz Ahmed Telwani. Hydatid Cyst of Spleen: A Diagnostic Challenge, North American Journal of Medical Science. 2013;5(1):10-20.

2. Rădulescu S, Mezer E. Parazitologie medicală. Ed. All. 1992;p.201-211.

3. Sunita Singh, Sonia Gulia, Pansi Gupta, et al. Isolated Splenic Hydatid Disease: A Rare Presentation. Journal Of Case Reports 2014;4(1):177180.

4. Tamara llić, Zsolt Becskei, Aleksandar Tasić, Sanda Dimitrijević. Followup study of prevalence and control of ascariasis in swine populations in Serbia. Acta Parasitologica. 2013,58(3),278-283.

5. Miller LA, Colby K, Manning SE, et al. Ascariasis in humans and pigs on small-scale farms. Maine, USA. 2010-2013. Emerg Infect Dis. 2015

6. Sedat Belli, Sami Akbulut1, Gurcan Erbay, Nazım Emrah Kocer. Spontaneous giant splenic hydatid cyst rupture causing fatal anaphylactic shock: A case report and brief literature review. The Turkish Journal of Gastroenterology. 2014;25:88-91.

7. Baraket Oussama, Moussa Makrem, Ayed Karim, et al. Surgical treatment and outcomes of hydatid cyst of the spleen. Open Journal of Gastroenterology. 2014;4,51-55.

8. M.M. Pukar and Shabari M. Pukar. Giant solitary hydatid cyst of spleen-A case report. Int J Surg Case Rep. 2013;4(4):435-437.

9. Shweta Rana, Shivani Kalhan, Sonia Hasija, Manmeet Kaur Gill, Neha Garg Jain. Primary Hydatid Cyst of Spleen: A rare entity. International Journal of Biomedical And Advance Research 2014; vol5,no8

10. S. Subramanyam, Metta Rajagopal, B. Venkata Rao, et al. "Primary Giant Hydatid Disease of The Spleen: A Rare Case Report with Review of Literature". Journal of Evidence based Medicine and Healthcare. 2015; Volume2,Issue8,Page:1103-1107.

11. Safak Ozturk, Mutlu Unver, Burcin Kibar ,et al. Isolated Retroperitoneal Hydatid Cyst Invading Splenic Hilum; Case Reports in Surgery. 2014

12. Gülsüm İclal Bayhan, Funda Çenesiz, Gönül Tanır, et al. First Case of Ascaris lumbricoides Infestation Complicated with Hemophagocytic Lymphohistiocytosis; Turkiye Parazitol Derg. 2015;39(2):164-6.

13. Himanshu Ajay Pendse, Ajita Jaywant Nawale, Sneha Satish Deshpande, Suleman Adam Merchant. Radiologic Features of Hydatid DiseaseThe Importance of Sonography. Journal of Ultrasound Medicine. 2015;34:895-905

14. Ayola A. Adegnika, Jeannot F. Zinsou, Saadou Issifou,et al. Randomized, Controlled, Assessor-Blind Clinical Trial To Assess the Efficacy of Singleversus Repeated-Dose Albendazole To Treat Ascaris lumbricoides, Trichuris trichiura, and Hookworm Infection. Antimicrob Agents Chemother. 2014;58(5):2535-2540. 